\title{
Connecting structure, dynamics and viscosity in sheared soft colloidal liquids: A medley of anisotropic fluctuations ${ }^{\dagger}$
}

\author{
Fabian Westermeier, ${ }^{* a}$ David Pennicard, ${ }^{b}$ Helmut Hirsemann, ${ }^{b}$ Ulrich H. Wagner, ${ }^{c}$ Christoph Rau, ${ }^{c \mathbb{I l}}$ Heinz Graafsma,${ }^{b \neq}$ \\ Peter Schall, ${ }^{d}$ M. Paul Lettinga, ${ }^{e}$ and Bernd Struth ${ }^{b}$
}

Structural distortion and relaxation are central to any liquid flow. Their full understanding requires simultaneous probing of the mechanical as well as structural and dynamical response. We provide the first full dynamical measurement of the transient structure using combined coherent $\mathrm{X}$-ray scattering and rheology on electrostatically interacting colloidal fluids. We find a stress overshoot during start-up of shear which is due to strong anisotropic overstretching and compression of nearest-neighbor distances. The rheological response is reflected in uncorrelated entropy-driven intensity fluctuations. While the structural distortion under steady shear is well described by Smoluchowski theory, we find an increase of the particle dynamics beyond the trivial contribution of flow. After cessation of shear, the full fluid microstructure and dynamics are restored, both on the structural relaxation timescale. We thus find unique structure-dynamics relations in liquid flow, responsible for the macroscopic rheological behavior of the system.

\section{Introduction}

The flow behavior of liquids and complex fluids is intimately connected to the adaptation of their structure. It is well known that many complex fluids dramatically change their mechanical properties when they are subjected to flow. One of the great challenges in fluid dynamics is to connect this transient material response to the underlying microstructure and dynamics. Such a connection is difficult to establish in conventional atomic liquids due to the involved small lengths and short time scales; this would require observation of the atomic structure and dynamics simultaneously during rheological measurements, and on time scales shorter than their flow relaxation time, a prohibitively difficult task for atomic liquids. We show here how probing the microscopic response of charged colloidal dispersions by high-flux coherent X-ray scattering during transient shear experiments gives unique insight in the mechanical response of liquids.

Suspensions of colloidal particles provide simple model systems for condensed matter as they can form liquid and crystal phases as well as gel and glass states by varying the concentration and interaction potential of the particles ${ }^{1-3}$. Each of these phases displays rich rheological behavior, covering wide ranges of non-Newtonian

\footnotetext{
${ }^{a}$ Max Planck Institute for the Structure and Dynamics of Matter, CFEL, Luruper Chaussee 149, 22761 Hamburg, Germany. E-mail: fabian.westermeier@mpsd.mpg.de

${ }^{b}$ Center for Free-Electron Laser Science, DESY, Notkestrasse 85, 22607 Hamburg, Germany.

${ }^{c}$ Diamond Light Source Ltd., Diamond House, Harwell Science and Innovation Campus, Didcot, Oxfordshire, OX11 ODE, United Kingdom.

${ }^{d}$ Van der Waals-Zeeman Institute, University of Amsterdam, POSTBUS 94485, 1090 GL Amsterdam, The Netherlands.

${ }^{e}$ Forschungszentrum Jülich, Institute of Complex Systems (ICS-3), 52425 Jülich, Germany.E-mail: p.lettinga@fz-juelich.de

$\ddagger$ Also at: Mid Sweden University, 85170 Sundsvall, Sweden.

I Also at: Department of Otolaryngology, Northwestern University Feinberg School of Medicine, Chicago, Illinois 60611, United States of America; School of Materials, University of Manchester, Grosvenor St., Manchester M1 7HS, United Kingdom.

$\S$ Also at: Laboratory for Soft Matter and Biophysics, KU Leuven, Celestijnenlaan 200D, B-3001 Leuven, Belgium.

$\dagger$ Electronic Supplementary Information (ESI) available: Additional information on the angular dependence of the intensity autocorrelation function and the shear dependent viscosity of PPG 4000.
}

responses 4,5 . The rheological behavior is central to applications of these suspensions in food, paints and cosmetics as well as to the function of biological systems. The ability to directly observe colloidal suspensions allows for direct test of theoretical descriptions of their flow and transient behavior ${ }^{6-8}$.

Although the fluid phase of colloidal hard spheres is conceptually the simplest phase, its rheology is still very complex ${ }^{9}$. In the regime of low shear rates, the viscosity decreases with increasing shear rate - the sample shows shear thinning - even at low volume fractions ${ }^{10}$. Yet, at high shear rates, strong shear thickening occurs due to hydro-clustering ${ }^{11-13}$. The distortion of the microstructure when convective forces dominate Brownian motion is thought to play a crucial role both in shear thinning and thickening. Steady-state shear distortion has been observed for a broad range of particle volume fractions and systems, in hard sphere systems ${ }^{14-21}$ as well as in charge-stabilized systems interacting via long range particle interactions ${ }^{18,21-23}$. A variety of methods has been used for these investigations, among them static light scattering ${ }^{14-16,23}$, neutron scattering ${ }^{17-19,24,25}$ and X-ray scattering $26-28$. During the last years, scanning confocal microscopy ${ }^{13,20,21}$ enabled the access to the three-dimensional structure of liquids under shear. The same was recently achieved by a combination of different sample geometries using neutron scattering 29,30 .

The structural distortions affect the shear-induced diffusion of the colloidal particles, hence coupling back to the flow dynamics. This effect is pronounced for colloidal glasses 7,31 , but is expected to be less pronounced for liquids, as the liquid phase is ergodic. On the other hand, a soft interaction potential may enhance structural distortions, as observed for soft glasses ${ }^{32-34}$. Clearly, the underlying dynamics of the particles also plays an important role and therefore, a pronounced coupling between rheology, microstructure and particle dynamics is to be expected. Connecting transient rheological behavior with the dynamically changing structure remains challenging even for this conceptually simple model system, despite its importance to many applications, and fundamental understanding of liquid flow.

In this paper we elucidate just this relation between transient 
rheology and adapting liquid structure, connecting these by dynamic structure factor measurements during rheological experiments. By performing coherent x-ray scattering experiments during start-up, steady shear and relaxation of a colloidal fluid, we reveal the dynamically changing microscopic structure and dynamics that underlie the complex rheological behavior. Using two different scattering geometries, we determine the full threedimensional structure factor revealing the strong shear-induced distortion of the nearest-neighbor structure. We find that the initial stress overshoot is caused by an overstretching and compression of particle distances, which increases nearest-neighbor potentials by several $k_{B} T$, with Boltzmann constant $k_{B}$ and absolute temperature $T$. The increase in stress is accompanied by a high-entropy state, signaled by enhanced intensity fluctuations. After cessation of shear, the driving force is the restoration of the compressed liquid structure to its quiescent configuration. These measurements for the first time give a full time-resolved, threedimensional picture of the distortion and relaxation of liquids driven far out of equilibrium.

\section{Experimental and Methods}

\subsection{Rheometer and Beamline Setup}

To probe the mechanical response simultaneously with the microscopic structure and dynamics, we employ a novel combination of rheology and dynamic $x$-ray scattering. This setup provides instantaneous structure factor measurement of the sheared suspension as reported in ${ }^{35-37}$, and is extended here to allow for a full structural and dynamical characterization in three dimensions. We exploit the high flux and high brilliance of third-generation synchrotron sources to record dynamically correlated data sets and determine the dynamic structure factor.

To use a commercial rheometer (MARS III, Thermo Fischer, Germany), a partially coherent X-ray beam was deflected by Germanium crystal optics by $90^{\circ}$, resulting in a vertical X-ray beam, parallel to the rotation axis of the rheometer. This beam passes through the layer of sheared suspension at around $14 \mathrm{~mm}$ to the rheometer axis. This setup is equivalent to the pilot installation at beamline BW1, DORIS III, DESY ${ }^{35}$. We employed two shear geometries: plate-plate and Couette geometry, both fabricated from polyimide (Vespel, DuPont) to reduce X-ray absorption at moderate X-ray energies. A gap opening of $0.8 \mathrm{~mm}$ was used in plateplate geometry. The Couette geometry consists of two concentric cylinders of respectively 30 and $28 \mathrm{~mm}$, so that the gap is $1.0 \mathrm{~mm}$, while the height of the cylinders is $3.0 \mathrm{~mm}$.

The measurements in Couette geometry were performed in the middle of the sample cell's gap. In plate-plate geometry the beam is directed along the gradient-of-velocity direction (in short gradient direction $\nabla \mathbf{v}$ ) so that structures that scatter in the flow direction $(\mathbf{v})$ or vorticity direction $(\nabla \times \mathbf{v})$ are probed. In the Couette geometry the beam is directed along the vorticity direction so that the flow-gradient plane is probed. Please note that the rheometer is stress-controlled and thus always moving slightly when switched on.

The use of two rheological geometries, a plate-plate and a Couette geometry ${ }^{38}$, allows probing the nearest-neighbor structure in three dimensions. We scale shear rates $\dot{\gamma}$ by the measured translational diffusion coefficient of a sphere of mean radius $R_{0}$ at infinite dilution $D_{0} \approx k_{B} T /\left(6 \pi \eta_{0} R_{0}\right)$ in a solvent with viscosity $\eta_{0}$, to define the Péclet number $P e=\dot{\gamma} R_{0}^{2} / D_{0}$. Structural distortions are expected to become pronounced for $P e>1$, when shear-induced motion dominates the diffusion and thermal motion can no longer equilibrate the structure.

The experiments were performed at beamline P10 of PETRA III at DESY, Germany ${ }^{39}$, at a photon energy of $8.05 \mathrm{keV}$ corresponding to a wavelength of $\lambda=0.15 \mathrm{~nm}$. To obtain a transversally coherent X-ray beam and a sufficient small speckle size in the detector plane, a set of slits upstream of the rheometer reduced the beamsize to typically $20 \times 20 \mu \mathrm{m}^{2}$ to $30 \times 30 \mu \mathrm{m}^{2}$ for coherent $\mathrm{X}$-ray scattering experiments. Additional experiments have been performed at beamline I13 of the Diamond Light Source (DLS), Didcot, UK, at the same photon energy and beamsize.

\subsection{X-ray detector: Lambda detector}

For the detection of anisotropic scattering patterns, a 2D photon counting detector is ideally suited, such as the novel LAMBDA detector, developed at DESY, Hamburg ${ }^{40}$. It is based on the Medipix3RX readout Application Specific Integrated Circuits ${ }^{41}$, bump bonded to $300 \mu \mathrm{m}$ Silicon sensor, which results in a high quantum efficiency close to $100 \%$. The LAMBDA satisfies the three main needs of a coherent scattering experiment: A sufficient small pixel size of $55 \times 55 \mu \mathrm{m}^{2}$ to spatially resolve speckle patterns, a high framing speed of up to 2000 frames/s with no time gap between consecutive frames to measure fast changes of the sample and a low noise level which is especially required if the framing rates of the detector are fast and thus the actually detected photons per pixel and frame are accordingly small.

The LAMBDA was placed at a distance $R_{\mathrm{det}} \approx 3.5 \mathrm{~m}$ (PETRA III) or $2.0 \mathrm{~m}$ (DLS) downstream of the sample position. The distance between rheometer and detector was bridged by a flight tube filled with Helium to minimize air scattering.

\subsection{Sample}

The colloidal fluid consists of almost monodisperse spherical silica particles suspended at a particle volume fraction of $\phi=$ 0.075 in poly-propylene glycol with an average molecular weight of $4000 \mathrm{~g} / \mathrm{mol}$ (PPG 4000) and a dynamic viscosity of $0.98 \mathrm{~Pa} \times \mathrm{s}$ at $25^{\circ} \mathrm{C}^{42}$. The $\mathrm{SiO}_{2}$-particles were prepared by poly-condensation of tetraethyl-orthosilicate $\left(\mathrm{Si}\left(\mathrm{OC}_{2} \mathrm{H}_{5}\right)_{4}\right)$ according to a Stöber synthesis ${ }^{43}$. In a second reaction step, the particles were coated with 3-trimethoxysilyl-propyl-methacrylate $\left(\mathrm{C}_{10} \mathrm{H}_{20} \mathrm{O}_{5} \mathrm{Si}\right)$. The mean particle radius was $R_{0}=105 \mathrm{~nm}$ with a particle size polydispersity of $\Delta R / R_{0}=5.2 \%$. The charged particles interact via long-range electrostatic repulsion. We determine an effective charge of $Z_{\text {eff }}=130 \mathrm{e}^{-}$and a Debye screening length $1 / \kappa=75 \mathrm{~nm}$ from excellent fits of the scattering data with the recently introduced Modified Penetrating Background-Corrected Rescaled Mean Spherical Approximation (MPB-RMSA) ${ }^{44,45}$. Thus, the effective radius of the particles is $R_{e f f}=180 \mathrm{~nm}$ and the effective volume fraction equals $\phi_{e f f}=0.38$, corresponding to an average particle-particle distance of $361 \mathrm{~nm}$. Hence, this is a very soft 
system.

\subsection{X-ray scattering experiments}

Steady shear rates in the range between $0.0005 \mathrm{~s}^{-1}$ and $20 \mathrm{~s}^{-1}$ have been probed. Scattering patterns were recorded with 10000 to 24000 frames per series. These series were always started 5 seconds before the rheometer started its motion and continued for 15 to 35 seconds after the rheometer stopped probing. To evaluate both the time evolution and the formation of anisotropy of the microstructure of the sample, the momentum transfer $Q$ dependent scattering intensity $I(Q)$ was extracted temporally and azimuthally resolved. 25 frames of $0.002 \mathrm{~s}$ and $0.01 \mathrm{~s}$ exposure time each (resulting in a time resolution of $0.05 \mathrm{~s}$ and $0.25 \mathrm{~s}$, respectively) were summed up for enhanced statistics, and $I(Q)$ was extracted for azimuthal slices of a width of $4^{\circ}$ and $10^{\circ}$, respectively.

The scattering intensity $I(Q)$ depends inter alia on the shape, size and size-distribution of the scatterers, given by the single particle form-factor $P(Q)$. For systems with direct particle interactions, $I(Q)$ is also modulated due to additional ordering of the particles, $I(Q) \propto P(Q) \times S(Q)$, where, $S(Q)$ is the static structure factor of the sample, which was subsequently derived from the scattering data by division with a scaled particle form factor. These $S(Q)$ were fitted using the MPB-RMSA model.

To evaluate overall intensity fluctuations in a region of interest (defined by a scattering vector segment $\Delta Q$ and an azimuthal angle segment $\Delta \theta$ ), the standard deviation of all recorded intensity values of 25 frames (corresponding to a time interval of $0.05 \mathrm{~s}$ to $0.25 \mathrm{~s}$ ) was calculated. This value, $\sigma(\Delta t, \Delta \theta, \Delta Q)$, was normalized by its temporal mean:

$$
\sigma_{\text {norm }}(\Delta t, \Delta \theta, \Delta Q)=\frac{\sigma(\Delta t, \Delta \theta, \Delta Q)}{\bar{\sigma}(\Delta \theta, \Delta Q)} .
$$

\subsection{X-ray Photon Correlation Spectroscopy in flow}

In X-ray Photon Correlation Spectroscopy (XPCS) ${ }^{46}$, the motion of scatterers leads to fluctuations of the scattered light intensity which is thus directly related to the particle dynamics via the intensity auto-correlation function

$$
g_{2}(Q, \tau)=\frac{\langle I(Q, t) I(Q, t+\tau)\rangle_{t}}{\langle I(Q, t)\rangle_{t}^{2}}
$$

where $\langle\ldots\rangle_{t}$ denotes a time average and $\tau$ is the lag time between different events 47,48 as shown previously for quiescent suspensions ${ }^{49,50}$. The application to sheared suspensions requires excellent stability of the rheology setup, and excellent time resolution. Here, the use of the new pixelated 2D photon counting detector, the LAMBDA ${ }^{40}$ is crucial, providing state-of-the-art temporal as well as spatial resolution.

Due to the intrinsic assumption (and subsequent calculation) of time averages, XPCS as a technique can only give access to mean sample dynamics for a certain time period. Therefore, to access changes of the sample dynamics with respect to the experimental time, intensity autocorrelation functions were calculated for different time intervals. While the interval length was $2 \mathrm{~s}$ (cor- responding to 1000 frames of $0.002 \mathrm{~s}$ exposure time), the time step between two subsequent intervals was $0.4 \mathrm{~s}$ (corresponding to 200 frames), thus resulting in a temporal overlap between two consecutive autocorrelation functions. Please note that due to the negligible scattering signal of the PPG 4000 polymers the scattering signal is strongly dominated by the colloidal particles and hence only the dynamics of the colloidal particles and not of the suspension medium PPG 4000 is accessed.

Recently XPCS has also been applied to measure local velocities in microfluidics ${ }^{51}$, which is important since for complex fluids also complex responses like shear banding should be anticipated $^{52}$. In the case of a fluid in laminar flow, the autocorrelation functions are determined by three factors: a) The diffusive motion of the scatterers due to thermal fluctuations in the sample, characterized by the relaxation rate $\Gamma$ which is related to the short-time diffusion coefficient $D(Q)$ by $\Gamma(Q)=D(Q) Q^{2}$; b) the transit time of the particles in the scattering volume, depending on the size of the scattering volume $h$ and the average velocity of the particles $v_{0}$, characterized by $v_{t} \propto v_{0} / h$; c) a shear induced decorrelation depending on the velocity gradient of the scatterers, characterized by the shear relaxation rate $\Gamma_{s}$ and depending on $Q$ and the average flow velocity $v_{0}$ via $\Gamma_{s}=Q_{\|} v_{0}$.

As the three factors determining the intensity autocorrelation function depend on the scattering geometry, they lead to ${ }^{53-55}$

$$
g_{2, \perp}(Q, \tau)=\beta \cdot \exp [-2 \Gamma \tau] \cdot \exp \left[-\left(v_{t} \tau\right)^{2}\right]+1
$$

and

$$
\begin{array}{r}
g_{2, \|}(Q, \tau, \dot{\gamma})=\beta \cdot \exp \left[-2 \Gamma \tau\left(1+\frac{(\dot{\gamma} \tau)^{2}}{3}\right)\right] \\
\cdot \exp \left[-\left(v_{t} \tau\right)^{2}\right] \cdot\left[\frac{\sin \left(\Gamma_{s} \tau\right)}{\Gamma_{s} \tau}\right]^{2}+1
\end{array}
$$

where $\beta$ is the experimental contrast of the autocorrelation function and $Q$ is the momentum transfer with the components $Q_{\|}$ parallel and $Q_{\perp}$ perpendicular to the flow direction. For details of the azimuthal dependence of the intensity autocorrelation function we refer to the ESI $\dagger$.

\subsection{Liquid structure distortion theory}

To analyze the distortion of the structure factor, we modeled $S(Q)$ with generalized Smoluchowski theory on the two-particle level, which neglects hydrodynamic interactions ${ }^{23,56}$. In particular, we followed equation (3) of ref. ${ }^{23}$

$$
\begin{array}{r}
S_{0}(Q, P e)=S^{\mathrm{eq}}(Q)+\frac{2}{P e Q_{\mathbf{v}}} \exp \left(\frac{2 Q_{\nabla \mathbf{v}}}{Q_{\mathbf{v}}} \frac{\left(Q_{\mathbf{v}}^{2}+\frac{1}{3} Q_{\nabla \mathbf{v}}^{2}+Q_{\nabla \times \mathbf{v}}^{2}\right)}{P e}\right) \\
\times \int_{Q_{\nabla \mathbf{v}}}^{ \pm \infty} \mathrm{d} C\left(Q_{\mathbf{v}}^{2}+C^{2}+Q_{\nabla \times \mathbf{v}}^{2}\right)\left[S^{\mathrm{eq}}\left(\left(Q_{\mathbf{v}}^{2}+C^{2}+Q_{\nabla \times \mathbf{v}}^{2}\right)^{1 / 2}\right)-S^{\mathrm{eq}}(Q)\right] \\
\times \exp \left(\frac{-2 C}{Q_{\mathbf{v}}} \frac{\left(Q_{\mathbf{v}}^{2}+\frac{1}{3} C^{2}+Q_{\nabla \times \mathbf{v}}^{2}\right)}{P e}\right) .
\end{array}
$$


Here, $Q_{\mathbf{v}}, Q_{\nabla \mathbf{v}}$, and $Q_{\nabla \times \mathbf{v}}$ are the magnitudes of the components of the scattering wavevector $Q$ along the flow, gradient, and vorticity direction, respectively and $S^{\text {eq }}$ is the static structure factor in equilibrium.

\section{Results and discussion}

\subsection{Microstructure under steady shear flow}

\subsubsection{Shear deformed microstructure.}

The colloidal fluid exhibits pronounced shear-thinning, as shown by the decreasing viscosity as a function of shear rate in Fig. 1b, while the pure suspension medium shows only marginal changes of the viscosity with flow (see ESI for details). Shear thinning sets in at $P e \geq 0.25$ and continues for higher shear rates until a Newtonian plateau at $P e \geq 20$ is reached. The X-ray measurements reveal a concomitant distortion of the nearest-neighbor structure as shown in Fig. 1a, left column. The figure combines both plateplate (top) and Couette geometries (bottom), allowing us to determine the full three-dimensional structure factor. With increasing shear rate, the structure factor becomes elliptically distorted in the flow-gradient plane for $P e<1$, with the long axis oriented along the compressional direction, as shown for $P e=0.58$ in Fig. 2a. At $P e>1$, in agreement with Ackerson ${ }^{22}$, two pairs of maxima emerge, indicating distinct two-particle configurations in the shear plane, as visible for $P e=5.76$. The corresponding real-space pair-distribution function $g(r)$ is shown in Fig. 2c and d, together with a probability plot of the most likely two-particle configurations, fig. 2e and $\mathrm{f}$.

The two pairs of distinct peaks indicate two co-existing orientational states: First, maxima and minima along the compressional and extensional axis form as the particle distance is compressed from an equilibrium distance of $350 \mathrm{~nm}$ to $335 \mathrm{~nm}$ due to the compressional component of shear flow, and stretched to $367 \mathrm{~nm}$ due to the dilatational component. Second, density fluctuations in the flow direction are mixed by convection, while this is not the case in the other two principal directions. Hence, the structure peak in the flow direction disappears while it remains mainly unaffected in the gradient and vorticity direction.

These trends are largely confirmed in theoretical modeling of the shear-distorted structure factor. We modeled the distortion of the structure factor with generalized Smoluchowski theory on the two-particle level, neglecting hydrodynamic interactions following Ref. ${ }^{23,56}$ (see Experimental and methods for details). The theoretical results are compared with the experimental structure factor in Fig. 1a (right column). Generally, the theory and experiments show good agreement. In hard sphere systems, the thermodynamic, Brownian stresses are dominating the hydrodynamic stresses in the shear thinning regime ${ }^{17,57}$, which seems to hold for strongly charged systems mainly interacting via long range repulsive interactions.

We can understand the compression of the particles quantitatively: Assuming an isotropic charge distribution, we estimate that the osmotically compressed particle distance gives rise to a force of $0.15 k_{B} T / \mathrm{nm}$ in the compression direction at $\mathrm{Pe}=$ 5.8. With the assumption of a linear flow profile between the two rheometer walls, this force is indeed of same order as the

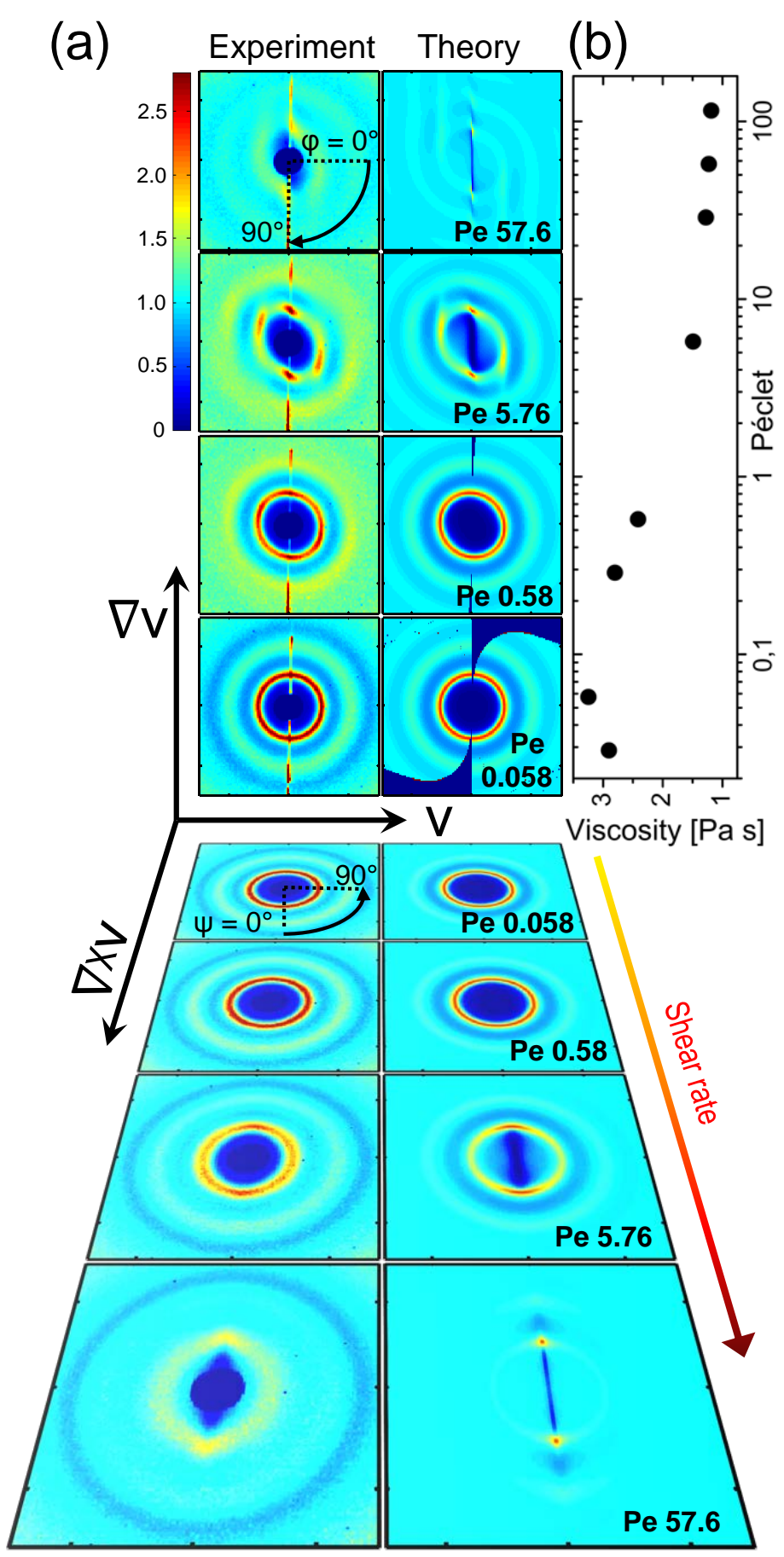

Fig. 1 Viscosity and static structure factors at steady shear. a) $S(Q)$ as measured (left columns) and as calculated using equation 5 (right columns) in the flow-gradient plane (Couette geometry, top 4 rows) and in the flow-vorticity plane (plate-plate geometry, bottom 4 rows, tilted) for $P e=0.058,0.58,5.76$ and 57.6 (bottom to top and in reverse order, respectively). The color-scale is identical for all images. Flow $\mathbf{v}$, gradient $\nabla \mathbf{v}$ and vorticity $\nabla \times \mathbf{v}$ direction are indicated by the coordinate system. The definition of the angle in the flow-gradient plane, $\varphi$, and in the flow-vorticity plane, $\psi$, are indicated in the top and fifth row, respectively. b) Viscosity as a function of Péclet number for values in between 0.0028 and 115.2 (corresponding to shear rates in between $0.005 \mathrm{~s}^{-1}$ and $\left.20 \mathrm{~s}^{-1}\right)$. 
(a)
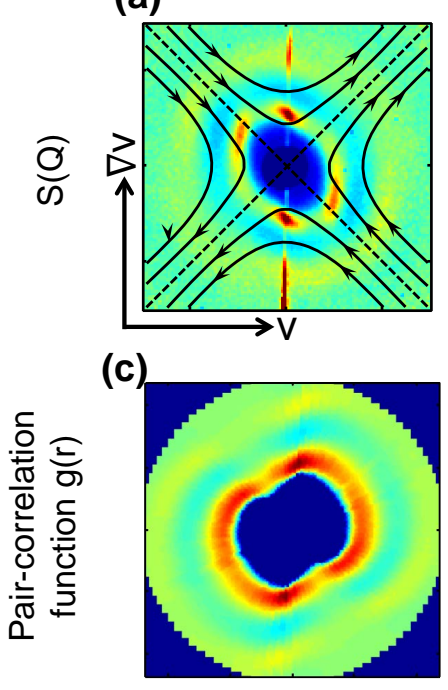

(e)

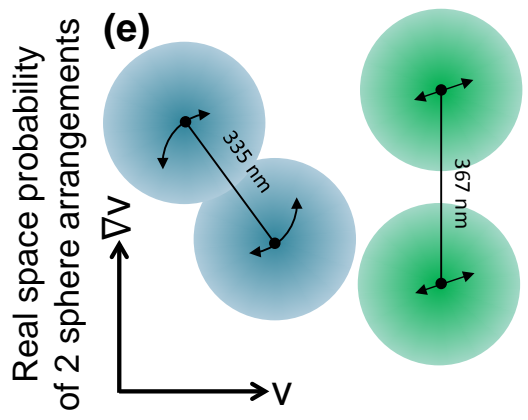

Flow-vorticity

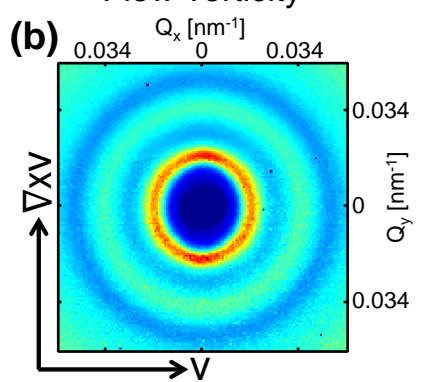

(d)

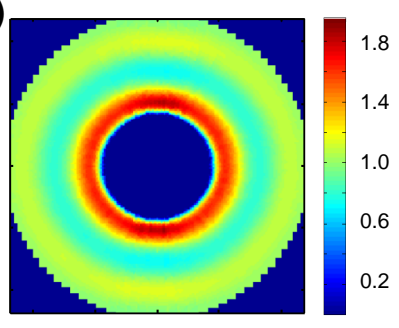

(f)

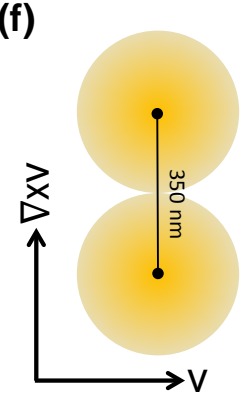

Fig. 2 Three-dimensional structure factor, pair correlation function and real-space representation at $P e=5.76$. Left column: Flow-gradient plane (Couette geometry); right column: Flow-vorticity plane (plate-plate geometry). a), b): Measured $S(Q)$ at $P e=5.76$, as also shown in figure 1, with a schematic representation of the extensional and compressional direction. c), d): Corresponding pair-correlation functions. e), f): Real space representation of the most probable two particle configurations in the particular plane.
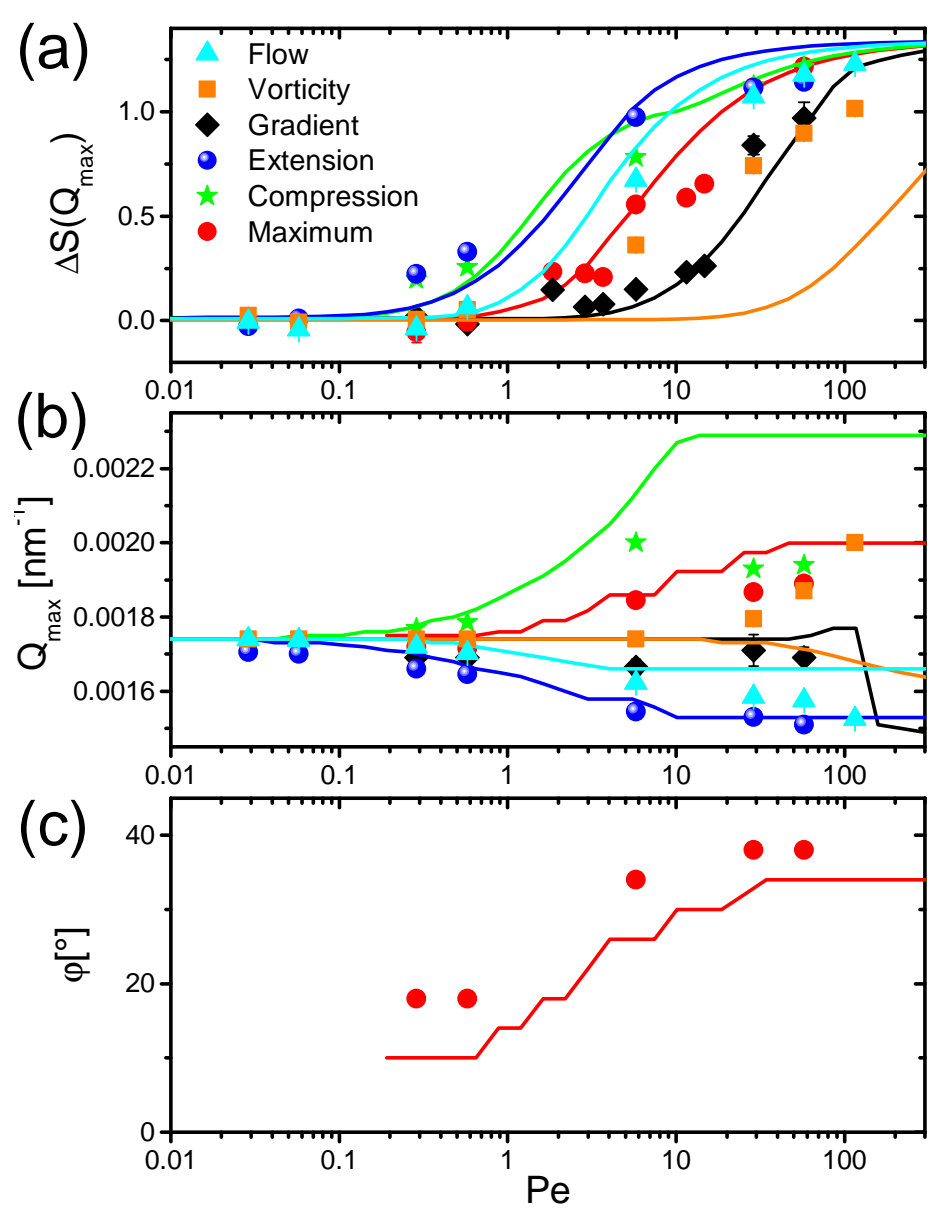

Fig. 3 Shear-rate dependence of the structural distortions. a) Relative height $\Delta S\left(Q_{\max }\right)=S^{\mathrm{eq}}\left(Q_{\max }\right)-S^{\text {shear }}\left(Q_{\max }\right)$; b) position $Q_{\max }$; and c) angular position $\varphi$ of the first maximum of the static structure factor $S(Q)$ for flow, gradient and vorticity direction, the direction of extensional and compressional flow and the maximum close to the compressional direction. The angular position in flow-gradient plane is defined in fig. $1 \mathrm{a}$, with respect to the flow direction.

Stokes drag between these two particles which we estimate to be $0.20 k_{B} T / \mathrm{nm}$, thus balancing the electrostatic force driving back the particles to their quiescent configuration.

\subsubsection{Shear rate dependence of the microstructural distor- tion.}

To compare these distortions in detail, we determine the excess equilibrium structure factor over that in steady shear, $\Delta S\left(Q_{\max }\right)=$ $S^{\text {eq }}\left(Q_{\max }\right)-S^{\text {shear }}\left(Q_{\max }\right)$, which we plot together with the peak position $Q_{\max }$ as a function of Péclet number in Fig. 3. Symbols indicate experimental data, and solid lines predictions by Smoluchowski theory. Both show consistently that deviations from the equilibrium structure start to emerge in the compression and dilation directions at $P e \approx 0.25$, precisely where shear thinning sets in. The corresponding dilation and compression of nearest-neighbor distances is clearly observed in the shift of $Q_{\max }$ along the respective directions. Both lead to an increase of $\Delta S\left(Q_{\max }\right)$ indicating the resulting enhanced structural distortion. Changes in the flow direction follow at $P e \approx 0.8$, showing a strong increase of $\Delta S\left(Q_{\max }\right)$, as predicted, and only a slight decrease in $Q_{\max }$. In 
the directions perpendicular to the flow (flow gradient and vorticity direction), changes occur only at the highest $P e$, accompanied by a slight decrease (vorticity) and increase (gradient) of the inter-particle distance. In the velocity-gradient plane, around $P e=0.5$, we observe the evolution of two distinct maxima pairs. With increasing shear rate one of the pairs rotates towards the compressional axis, see Fig. 3c. This indicates that indeed the microstructure is strongly compressed, compared to the equilibrium structure depicted in fig. 2a. Discrepancy between theory and experiment occurs in the vorticity direction, where $\Delta S\left(Q_{\max }\right)$ increases at much lower Pe than predicted, and inter-particle distances decrease rather than increase as predicted by theory (cf. orange symbols and line). These deviations in the vorticity direction hint at hydrodynamic interactions, which are not included in the theory, but can induce isotropic distortions ${ }^{58}$. As lubrication hydrodynamics and thus hydro-clustering are strongly suppressed $^{9,12,59}$ due to the long-range repulsive potential and the low volume fraction of the sample system, mainly far-field hydrodynamic interactions should contribute to the distortion. Another reason might be a possible limitation of the model to small departures from equilibrium.

In contrast to concentrated hard sphere systems, we do not detect an increase of $\Delta S\left(Q_{\max }\right)$ in the flow direction ${ }^{15,25,36}$. Apparently, in the probed electrostatically interacting systems a compression does not lead to an enhancement of structure. This can be attributed to the fact that in hard sphere systems structure arises due to the collision of nano-particles with each other, while this is strongly diminished in electrostatically interacting systems where the particles tend to maximize the distance between each other. As the potential is softer and particles have more room to adjust, one expects more pronounced flow-induced changes from the equilibrium structure factor. Indeed, around medium $\mathrm{Pe}$ in between 1 to 10 the structure is strongly diminished, especially in comparison to hard sphere systems ${ }^{19,30}$. This could also explain the relatively low Péclet numbers where we first observe structural changes as compared to hard sphere experiments $21,30,60$. Note also that the theory is only valid for low Péclet numbers, so that the correspondence of theory and experiments for $P e \gg 1$, such as the merging of the maxima in the flow-gradient plane with increasing Péclet numbers, may be fortuitous.

\subsection{Transient behavior}

Our measurements hence reveal the pronounced distortion of the nearest-neighbor structure underlying the rheological shear thinning. We can now use the full time resolution to follow dynamic behavior during the transients. To elucidate the build-up and relaxation of the distorted structure, we follow the system during start-up, steady-state, and after cessation of shear, as shown in Fig. 4.

\subsubsection{Transients in rheology and microstructure.}

The rheology already reveals distinct transient behavior during start-up, as shown by the viscosity evolution before reaching steady-state in Fig. 4, d to f. For $P e<0.1$ (d), the viscosity monotonically increases before reaching its plateau value, while for higher Péclet numbers (e and $\mathrm{f}$ ), it overshoots at strains $\gamma \approx 0.5$, before reaching the plateau. Interestingly, this overshoot is directly reflected in the microstructure: For $P e=0.576$, the peak of the structure factor grows, while the particle distances adjust to their overstretched steady-state flow configuration as shown by the concomitant decrease of $Q_{\max }$ in $\mathrm{g}-\mathrm{i}$ of Fig. 4). This overshoot of the structure factor indicates enhanced positional correlation of the strained structure in the flow direction, suggesting elastic straining effects as repulsive particles are squeezed together during start-up of shear.

A new steady-state structure with decreased inter-particle correlations is established about $10 \mathrm{~s}$ after the start of shear. Interestingly, the transient behavior is also reflected in the fluctuations of the scattered intensity $\sigma_{\text {norm }}(\Delta t, \Delta \theta, \Delta Q)$ defined by equation (1) (see experimental and methods), as shown in Fig. 4e. The fluctuations were recorded at $Q=0.015 \mathrm{~nm}^{-1}$, near $Q_{\max }$ at the slope of the first maximum of $S(Q)$. These enhanced fluctuations indicate higher entropy of the system: In equilibrium, the system is isotropic, while out of equilibrium, when stress is applied, the symmetry is broken as the sample evolves into an anisotropic oriented state. The more pronounced intensity fluctuations during the transient can be seen as the footprint of a different entropic state during reorganization of the colloidal system. For $P e=5.8$ all responses are more pronounced, showing damped oscillations reminiscent of an elastic response, where the system swings around its new steady state structure, while for $P e<0.1$, where Brownian motion is dominating, no deformation is observed as the system can counteract deformation.

After cessation of flow, all parameters relax to their equilibrium value within about 10 seconds both for $P e=0.576$ and $P e=5.76$. This relaxation time is on the order of the structural relaxation time $\tau_{S}=4 R_{0}^{2} / D_{0}=20 \mathrm{~s}$. The relaxation time is independent of the previous applied shear rate, the only difference being the amplitude of relaxation, which is highest for $P e=5.76$ where the sheared state is furthest from the quiescent state. At lower Péclet number these transient structural effects vanish, as shown for $P e=0.0576$, for which the smooth evolution of viscosity is reflected in an unaffected micro-structure. This decrease of the stress overshoot with decreasing shear rate has been also observed in hard sphere systems ${ }^{61-63}$.

\subsubsection{Transients in the dynamics.}

The unique time resolution of our setup allows us to follow the suspension dynamics during the transients. To do so, we computed autocorrelation functions of the scattered intensity for short time intervals of two seconds to evaluate particle dynamics; sliding time intervals then allowed us to follow changes of particle dynamics during the transients. The resulting evolution of the autocorrelation function is shown in a color-coded representation in Fig. 5a, taken at $Q_{\max }$, the maximum of $S(Q)$ in flow direction. Experimental time, plotted along the horizontal axis, again demarcates start-up shear $(t \approx 5 \mathrm{~s})$, steady-state shear $(5 \mathrm{~s}<t<30 \mathrm{~s})$, and relaxation $(t>30 \mathrm{~s})$. The relaxation time scale $\tau$ plotted vertically indicates the evolution of the dynamics. Clearly, when shear is applied, the autocorrelation function decays faster, indicating enhanced diffusion and faster particle dynamics. During steadystate shear, the corresponding autocorrelation functions can be 
Pe 0.058
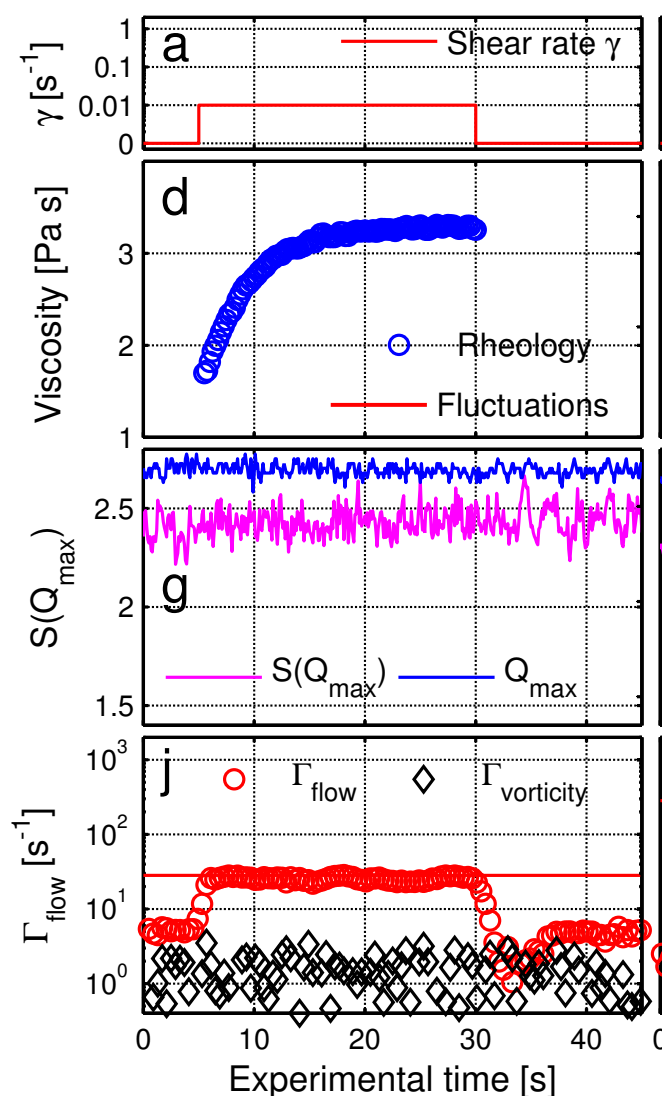

Pe 0.576
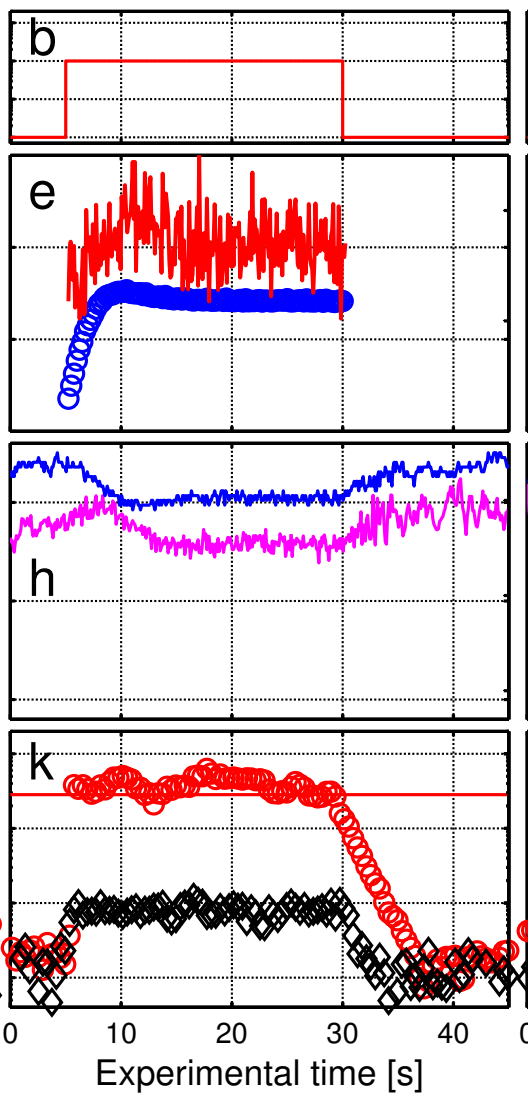

Pe 5.762
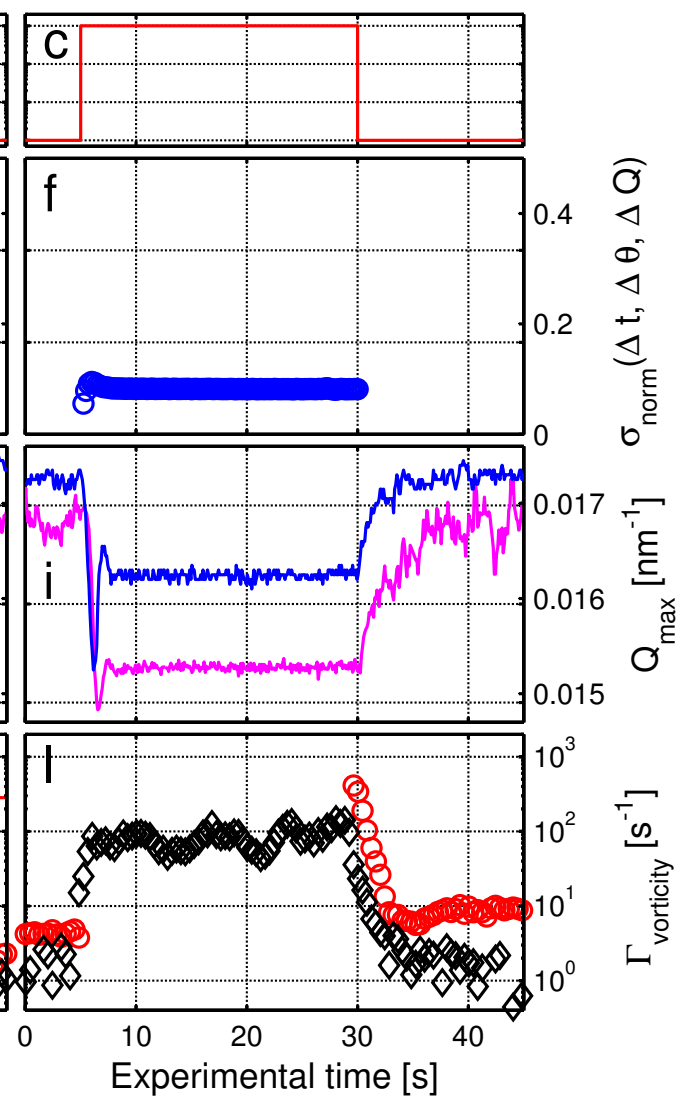

Fig. 4 Transients at $P e=0.058,0.576$ and 5.762. a) - c): Applied shear-rate $\dot{\gamma}$. d) - f): Temporal evolution of the viscosity (blue circles) and for $P e=0.576$ fluctuations of the scattering intensity $I(Q)$ (red solid line) at $Q=0.015 \mathrm{~nm}^{-1}$. g) - i): Temporal evolution of $S\left(Q_{\text {max }}\right)$ (pink line) and the

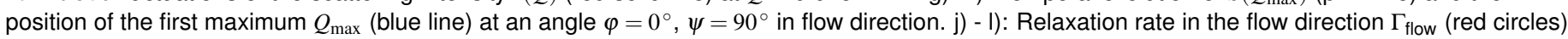

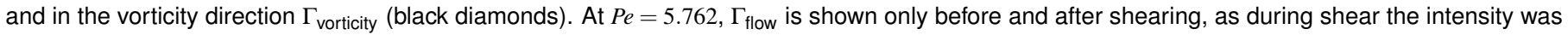
decorrelating too fast for reliable dynamics measurements. The solid red lines correspond to the expected relaxation rates assuming a linear flow

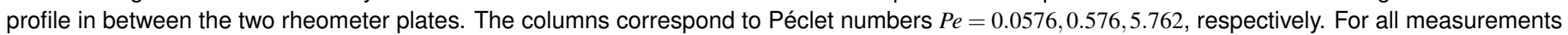
the rheometer started after $5.0 \mathrm{~s}$ and sheared for $25 \mathrm{~s}$. 


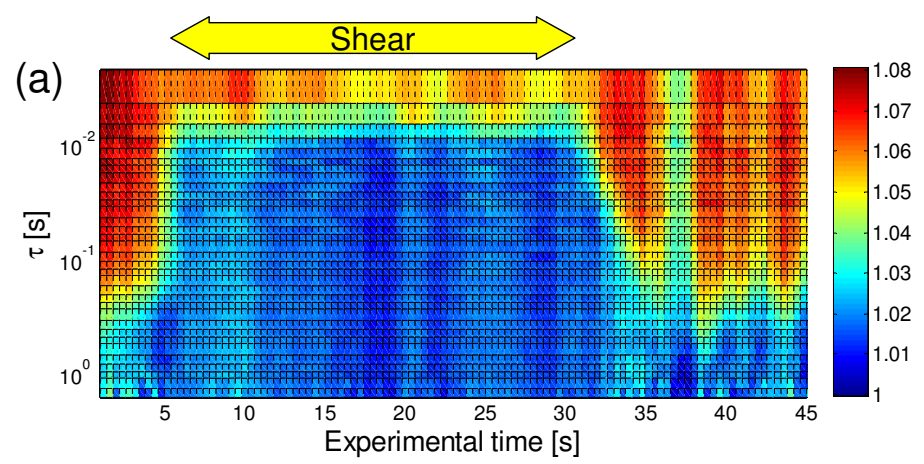

nicely modeled assuming an additional shear relaxation rate $\Gamma_{s}$ of individual particles in the scattering volume (see experimental and methods, equations (3) and (4)). The flow velocity calculated from the extracted $\Gamma_{s}$ agrees exactly with the applied shear rate when assuming a linear flow profile in between the two rheometer plates, as can be appreciated comparing Fig. $4 \mathrm{j}$ and $\mathrm{k}$, red solid line and red circles.

During start-up, the autocorrelation function shows two distinct decays as depicted in Fig. 5b, indicating the presence of two relaxation processes, thermal (before the onset of shear) and shear induced (after the onset of shear). Assuming that shear-induced relaxation with rate $\Gamma_{s}$ superimposes on the thermal relaxation of the quiescent suspension with rate $\Gamma$, the decay of the autocorrelation function can be described by

$$
g_{2}(\tau, Q)=\beta\left((1-b) \exp [-2 \Gamma \tau]+b\left(\frac{\sin \left(\Gamma_{s} \cdot \tau\right)}{\Gamma_{s} \cdot \tau}\right)^{2}\right)+1
$$

From fits (solid lines in Fig 5b) to the measured decays, we find that the weighting factor $b$ of the shear-induced contribution increases linearly with time after start of shear, as shown in the inset of Fig. 5b. It thus increases with the ratio of frames (for the calculation of the autocorrelation function) before and after onset of shear. As an example, the autocorrelation function for the time interval from $4.0 \mathrm{~s}$ to $6.0 \mathrm{~s}$, where approximately half of the frames for the calculation of the $g_{2}$-function are measured before and the other half measured after the onset of shear flow, gives a value of $b=0.42$. For the last point, obtained for a correlation time interval entirely during shearing, the weighting factor is indeed close to 1 , corresponding to the motion of all particles with the velocity of shear flow. We thus conclude that the flow is transmitted instantaneously throughout the gap of the shear cell.

The particle relaxation after cessation of flow is characterized by a gradual shift of the autocorrelation functions towards a slower decay, as is apparent for $t>30 \mathrm{~s}$ in Fig. 5a. To compare the autocorrelation functions at all experimental times, we modeled them with the Kohlrausch-Williams-Watts function ${ }^{64}$, $g_{2}(Q)=\beta \cdot \exp \left(-2 \cdot(\Gamma \tau)^{\gamma}\right)+1$, with $\gamma$ being the exponent of the exponential decay. The fits nicely agree with the experimental autocorrelation functions during cessation of shear, indicating a broad distribution of decay times and a gradual recovery of the equilibrium relaxation time, as depicted in Fig. 5c. The extracted relaxation rates $\Gamma$ in flow and vorticity direction are depicted in Fig. $4 \mathrm{j}-1$, together with the structural changes in panels $\mathrm{g}-\mathrm{i}$. For a low Péclet number of 0.0576 , no changes of $\Gamma$ can be detected perpendicular to the flow direction during start up nor after cessation of shear, indicating that there is almost no contribution of shear to the thermal relaxation rate due to diffusion. This situation changes for increasing shear rates $P e>0.1$, where the relaxation rate increases due to the applied shear. The relaxation process after cessation of shear takes place during approximately $10 \mathrm{~s}$, corresponding to the timescale of the relaxation of the microstructure, which is on the order of the structural relaxation time $\tau_{S}$. Hence, the time scale of the dynamic relaxation coincides nicely with the time scale of structural relaxation, confirming the tight relationship between microstructure and dynam- 
ics. Because of this coupling, the dynamics continues to be fast, even after complete cessation of shear, while the original quiescent structure has not reformed, until the original structure has re-established, and concomitantly, the dynamics have decayed to its original rate. The initial decay rate during the recovery of the equilibrium relaxation time is about one to two orders of magnitude higher than the quiescent diffusion, indicating thus that there is a driving force for the reestablishment of the equilibrium microstructure. This driving force is given by the difference between the potential at the non-equilibrium compressed state and the potential in the liquid quiescent state, where the colloidal system balances the forces between individual particles and thus restores the initial microstructure. Moreover, the decay of overall fluctuations, comprising both correlated and uncorrelated ones, can be seen as an indication of energy release from the system.

\section{Summary and conclusion}

Our combined measurements reveal the three-dimensional structure and dynamics underlying the rheology during start-up, steady shear and relaxation of charged colloidal liquids. The shear thinning of the colloidal fluid can be attributed to the overall distortion of the structure factor. We confirm predictions by generalized Smoluchowski theory ${ }^{56}$ for the distortion of the full three-dimensional structure factor in steady shear flow which leads to shear thinning. In particular, the flow gradient plane gives us access to the compressional and extensional direction where the structure is compressed and stretched, respectively. This allows us to estimate the forces to compress the particles. We show that the compression force is sufficient to overcome the electrostatic potential inducing the microstructure in the quiescent liquid.

The compression forces form the basis for understanding the transient behavior. The structural restoration after cessation of shear flow is accompanied by a pronounced slowing down of the colloidal dynamics which is initially up to two orders of magnitude higher than the quiescent diffusion. This anomalous flow-enhanced diffusion manifests directly the electrostatic driven restoring force: it tends to equilibrate the particle concentration in the compression and vorticity direction, while filling the depleted space in the extensional and flow direction on microscopic length scales corresponding to $Q_{\max }$. We suggest that this is in analogy to collective diffusion in equilibrium, where the osmotic compressibility $\frac{\partial n}{\partial \Pi}=S(0) /\left(k_{B} T\right)$ drives the diffusion for $Q \rightarrow 0$ for high-density gradients. It is shown theoretically that this effect is enhanced by shear flow ${ }^{65-67}$, which is confirmed by simulations of fluidized soft glassy systems ${ }^{32,34}$, where unbalanced contact forces drive the system back to its equilibrium state. In analogy, the long-range repulsive interactions between the charged particles likely enhances this process and makes it occur more pronounced than is found for short-ranged repulsive systems, an effect which prompts for more theoretical work.

Stress relaxation after cessation of shear flow was also observed for soft glasses and related to the restoration of structure in simulations ${ }^{33}$. We show here experimentally that indeed the stress relaxation is governed by the structural and dynamical relaxation in the fluid phase, which takes place on the timescales of the struc- tural relaxation time.

In addition, entropic intensity fluctuations, quantified by $\sigma_{\text {norm }}(\Delta t, \Delta \theta, \Delta Q)$, show a similar transient behavior as both the stress and the microstructure. Due to the breaking of symmetry when shear is applied, the system can evolve towards more possible configurations, pointing towards higher entropy of the system reflected by the increased fluctuations. The events that cause the fluctuations persist in these soft systems, because of their regenerative character. This is consistent with the enhanced diffusion as found by XPCS.

In conclusion, the combination of rheology and high-flux coherent X-ray scattering on soft colloidal dispersion allowed us to identify stress overshoots in transient with overshoots in the structure deformation as well as in entropic intensity fluctuations. These observations reveal the connection between microscopic structure and dynamics and the mechanical response of fluids. Moreover, we could show that shear flow strongly enhances the diffusion, which returns to the equilibrium behavior at the same time scale as the equilibrium structure is restored after cessation of shear flow. We argue that the unique combination of experiments we put forward here opens the road for the full understanding of complex flow behavior on all relevant length-scales.

\section{Acknowledgements}

We wish to acknowledge the support of the beamline staff of P10, in particular Michael Sprung, and the support of the beamline staff of I13, in particular Andrew Peach. We would like to thank Dmitry Denisov and Dang Minh Triet for fruitful discussions. For the Lambda development, we would like to thank Sergej Smoljanin. We are grateful to Jan Dhont and Gerhard Nägele for helpful suggestions and discussions. 


\section{References}

1 P. N. Pusey and W. van Megen, Nature, 1986, 320, 340-342.

2 P. J. Lu and D. A. Weitz, Annual Review of Condensed Matter Physics, 2013, 4, 217-233.

3 V. J. Anderson and H. N. W. Lekkerkerker, Nature, 2002, 416, 811-815.

4 J. Vermant and M. J. Solomon, Journal of Physics-Condensed Matter, 2005, 17, R187-R216.

5 D. Vlassopoulos and M. Cloitre, Curr. Opin. Colloid Interface Sci., 2014, 19, 561-574.

6 J. M. Brader, Journal of Physics: Condensed Matter, 2010, 22, 363101.

7 M. Laurati, K. J. Mutch, N. Koumakis, J. Zausch, C. P. Amann, A. B. Schofield, G. Petekidis, J. F. Brady, J. Horbach, M. Fuchs and S. U. Egelhaaf, Journal of Physics-Condensed Matter, 2012, 24, 464104.

8 E. Nazockdast and J. F. Morris, Soft Matter, 2012, 8, 42234234.

9 H. M. Laun, Angewandte Makromolekulare Chemie, 1984, 123, 335-359.

10 D. R. Foss and J. F. Brady, Journal of Rheology, 2000, 44, 629651.

11 D. R. Foss and J. F. Brady, Journal of Fluid Mechanics, 2000, 407, 167-200.

12 N. J. Wagner and J. F. Brady, Physics Today, 2009, 62, 27-32.

13 X. Cheng, J. H. McCoy, J. N. Israelachvili and I. Cohen, Science, 2011, 333, 1276-1279.

14 B. J. Ackerson, J. van der Werff and C. G. de Kruif, Phys. Rev. A, 1988, 37, 4819-4827.

15 B. J. Ackerson, C. G. De Kruif, N. J. Wagner and W. B. Russel, The Journal of Chemical Physics, 1989, 90, 3250-3253.

16 N. J. Wagner and W. B. Russel, Physics of Fluids A, 1990, 2, 491-502.

17 J. W. Bender and N. J. Wagner, Journal of Colloid and Interface Science, 1995, 172, 171-184.

18 B. J. Maranzano and N. J. Wagner, The Journal of Chemical Physics, 2002, 117, 10291-10302.

19 D. P. Kalman and N. J. Wagner, Rheologica Acta, 2009, 48, 897-908.

20 X. Cheng, X. Xu, S. A. Rice, A. R. Dinner and I. Cohen, Proceedings of the National Academy of Sciences, 2012, 109, 63-67.

21 B. Xu and J. F. Gilchrist, The Journal of Chemical Physics, 2014, 140, 204903.

22 B. J. Ackerson, Physica A: Statistical Mechanics and its Applications, 1991, 174, 15-30.

23 Y. Yan and J. Dhont, Physica A: Statistical Mechanics and its Applications, 1993, 198, 78-107.

24 B. J. Ackerson, J. B. Hayter, N. A. Clark and L. Cotter, The Journal of Chemical Physics, 1986, 84, 2344-2349.

25 H. Watanabe, M.-L. Yao, K. Osaki, T. Shikata, H. Niwa, Y. Morishima, N. P. Balsara and H. Wang, Rheologica Acta, 1998, 37, 1-6.

26 H. Hoekstra, J. Mewis, T. Narayanan, and J. Vermant, Lang- muir, 2005, 21, 11017-11025.

27 H. Versmold, S. Musa, , C. Dux, P. Lindner and V. Urban, Langmuir, 2001, 17, 6812-6815.

28 H. Versmold, S. Musa and A. Bierbaum, The Journal of Chemical Physics, 2002, 116, 2658-2662.

29 A. K. Gurnon, C. R. Lopez-Barron, A. P. R. Eberle, L. Porcar and N. J. Wagner, Soft Matter, 2014, 10, 2889-2898.

30 A. K. Gurnon and N. J. Wagner, Journal of Fluid Mechanics, 2015, 769, 242-276.

31 V. Chikkadi, D. M. Miedema, M. T. Dang, B. Nienhuis and P. Schall, Phys. Rev. Lett., 2014, 113, 208301.

32 J. R. Seth, L. Mohan, C. Locatelli-Champagne, M. Cloitre and R. T. Bonnecaze, Nat Mater, 2011, 10, 838-843.

33 L. Mohan, R. T. Bonnecaze and M. Cloitre, Physical Review Letters, 2013, 111, 268301.

34 L. Mohan, C. Pellet, M. Cloitre and R. Bonnecaze, Journal of Rheology, 2013, 57, 1023-1046.

35 B. Struth, K. Hyun, E. Kats, T. Meins, M. Walther, M. Wilhelm and G. Grübel, Langmuir, 2011, 27, 2880-2887.

36 D. Denisov, M. Dang, B. Struth, G. Wegdam and P. Schall, Scientific Reports, 2013, 3, 1631.

37 M. P. Lettinga, P. Holmqvist, P. Ballesta, S. Rogers, D. Kleshchanok and B. Struth, Phys. Rev. Lett., 2012, 109, 246001.

38 J. M. Kim, A. P. R. Eberle, A. K. Gurnon, L. Porcar and N. J. Wagner, Journal of Rheology, 2014, 58, 1301-1328.

39 A. V. Zozulya, S. Bondarenko, A. Schavkan, F. Westermeier, G. Grübel and M. Sprung, Opt. Express, 2012, 20, 1896718976.

40 D. Pennicard, S. Lange, S. Smoljanin, H. Hirsemann and H. Graafsma, Journal of Instrumentation, 2012, 7, C11009.

41 R. Ballabriga, J. Alozy, G. Blaj, M. Campbell, M. Fiederle, E. Frojdh, E. H. M. Heijne, X. Llopart, M. Pichotka, S. Procz, L. Tlustos and W. Wong, Journal of Instrumentation, 2013, 8, C02016.

42 M. G. McLin and C. A. Angell, The Journal of Physical Chemistry, 1991, 95, 9464-9469.

43 W. Stöber, A. Fink and E. Bohn, J. Coll Inter. Sci., 1968, 26, 62-69.

44 M. Heinen, P. Holmqvist, A. J. Banchio and G. Nägele, J. Chem. Phys., 2011, 134, 044532.

45 M. Heinen, P. Holmqvist, A. J. Banchio and G. Nägele, J. Chem. Phys., 2011, 134, 129901.

46 G. Grübel and F. Zontone, J. Alloy Comp., 2004, 362, 3-11.

47 M. Sutton, S. G. J. Mochrie, T. Greytak, S. E. Nagler, L. E. Berman, G. A. Held and G. B. Stephenson, Nature, 1991, 352, 608-610.

48 T. Thurn-Albrecht, W. Steffen, A. Patkowski, G. Meier, E. W. Fischer, G. Grübel and D. L. Abernathy, Phys. Rev. Lett., 1996, 77, 5437-5440.

49 J. Gapinski, A. Patkowski, A. J. Banchio, J. Buitenhuis, P. Holmqvist, M. P. Lettinga, G. Meier and G. Nagele, The Journal of Chemical Physics, 2009, 130, 084503.

50 F. Westermeier, B. Fischer, W. Roseker, G. Grübel, G. Nägele 
and M. Heinen, Journal of Chemical Physics, 2012, 137, 114504.

51 A. Fluerasu, P. Kwasniewski, C. Caronna, F. Destremaut, J.B. Salmon and A. Madsen, New Journal of Physics, 2010, 12, 035023.

52 J. K. G. Dhont and W. J. Briels, Rheo. Acta, 2008, 47, 257-281.

53 D. P. Chowdhury, C. M. Sorensen, T. W. Taylor, J. F. Merklin and T. W. Lester, Appl. Opt., 1984, 23, 4149-4154.

54 S. Busch, T. Jensen, Y. Chushkin and A. Fluerasu, The European Physical Journal E: Soft Matter and Biological Physics, 2008, 26, 55-62.

55 W. R. Burghardt, M. Sikorski, A. R. Sandy and S. Narayanan, Phys. Rev. E, 2012, 85, 021402.

56 J. K. G. Dhont, Journal of Fluid Mechanics, 1989, 204, 421431.

57 N. J. Wagner and B. J. Ackerson, The Journal of Chemical Physics, 1992, 97, 1473-1483.

58 M. P. Lettinga, H. Wang and J. K. G. Dhont, Physical Review E, 2004, 70, 061405.

59 J. Bergenholtz, J. F. Brady and M. Vicic, Journal of Fluid Me- chanics, 2002, 456, 239-275.

60 M. C. Newstein, H. Wang, N. P. Balsara, A. A. Lefebvre, Y. Shnidman, H. Watanabe, K. Osaki, T. Shikata, H. Niwa and Y. Morishima, The Journal of Chemical Physics, 1999, 111, 4827-4838.

61 H. Watanabe, M.-L. Yao, A. Yamagishi, K. Osaki, T. Shitata, H. Niwa and Y. Morishima, Rheologica Acta, 1996, 35, 433445.

62 C. P. Amann, M. Siebenbürger, M. Krüger, F. Weysser, M. Ballauff and M. Fuchs, Journal of Rheology, 2013, 57, 149-175.

63 N. Koumakis, A. Pamvouxoglou, A. S. Poulos and G. Petekidis, Soft Matter, 2012, 8, 4271-4284.

64 G. Williams and D. C. Watts, Trans. Faraday Soc., 1970, 66, 80-85.

65 M. Marchioro and A. Acrivos, Journal of Fluid Mechanics, 2001, 443, 101-128.

66 A. M. Leshansky and J. F. Brady, Journal of Fluid Mechanics, 2005, 527, 141-169.

67 A. M. Leshansky, J. F. Morris and J. F. Brady, Journal of Fluid Mechanics, 2008, 597, 305-341. 